\title{
A fingerprint identification algorithm by clustering similarity
}

\author{
TIAN Jie, HE Yuliang, CHEN Hong \& YANG Xin \\ Center for Biometric Research and Testing (CBRT), Key Laboratory of Complex Systems and Intelligence \\ Science, Institute of Automation, Chinese Academy of Sciences, Graduate School of the Chinese Acad- \\ emy of Sciences, Beijing 100080, China \\ Correspondence should be addressed to Tian Jie (email: tian@doctor.com; jie.tian@mail.ia.ac.cn) \\ Received November 30, 2004
}

\begin{abstract}
This paper introduces a fingerprint identification algorithm by clustering similarity with the view to overcome the dilemmas encountered in fingerprint identification. To decrease multi-spectrum noises in a fingerprint, we first use a dyadic scale space (DSS) method for image enhancement. The second step describes the relative features among minutiae by building a minutia-simplex which contains a pair of minutiae and their local associated ridge information, with its transformation-variant and invariant relative features applied for comprehensive similarity measurement and for parameter estimation respectively. The clustering method is employed to estimate the transformation space. Finally, multi-resolution technique is used to find an optimal transformation model for getting the maximal mutual information between the input and the template features. The experimental results including the performance evaluation by the 2 nd International Verification Competition in 2002 (FVC2002), over the four fingerprint databases of FVC2002 indicate that our method is promising in an automatic fingerprint identification system (AFIS).
\end{abstract}

Keywords: dyadic scale space (DSS), minutia-simplex, multi-resolution, comprehensive similarity.

DOI: 10.1360/04yf0113

\section{Introduction}

Automatic fingerprint identification system (AFIS) has been widely used and it consists of fingerprint enhancement and matching. Fingerprint enhancement is to recover the topology structure of ridges and valleys from the corrupted image during capture because of impression, skin, and reader etc. Most of fingerprint enhancement algorithms are based on orientation field estimation, such as Lin et al.'s ${ }^{[1]}$ Gabor filter method. These methods have a defect that a local bad-quality region needs to be enhanced while estimating its local orientation field. As a result, the orientation field is unreliable and the enhancement is compromised.

Fingerprint matching is to obtain the maximal mutual information between the input 
and the template features. It generally consists of extracting feature, modelling relative structure, alignment, and therefore obtaining the maximal similarity between two fingerprints. Fingerprint matching involves a wide range of algorithms with different techniques. These schemes are based predominantly on local landmarks, exclusive global features as well as comprehensive fingerprint features. The minutiae-based matching methods ${ }^{[2,3]}$ are widely used in memory-limited AFISs because they need less memory expense and having time saving advantages. However, minutiae cannot characterize overall pattern of a fingerprint, and it is hard to further improve their performance. The exclusive global feature-based techniques ${ }^{[4,5]}$ match the global patterns of the fingerprint texture by aligning the input global features and calculating the maximum mutual global information between two fingerprints. In these methods, however, the central point should be determined with a reliable accuracy and it is difficult to deal with distortion in fingerprints. Comprehensive feature-based techniques ${ }^{[6,7]}$ are also seen as a hybrid matching method by fusing minutiae, local features and global features. Local features help accelerate the alignment of the unregistered minutia patterns in different sizes. Global features are used to overcome the shortness of minutiae and local features in bad quality fingerprints. With the reasonable time and memory expenses, comprehensive feature-based techniques outperform the above mentioned matching methods. In addition, they combine various classifiers for fingerprint matching. With the advanced hardware technology, these approaches have become popular for their good performance in acceptable memory expense in recent years.

This paper introduces an identification algorithm by clustering similarity with the view to overcome the dilemmas encountered in fingerprint matching process. We first employed Cheng et al.'s dyadic scale space (DSS)-based enhancement $\operatorname{method}^{[8]}$ for a fingerprint is often affected by multi-spectrum noises. Minutiae and local ridge information are combined, and minutia-simplex is built to describe a 2nd order relative structure among minutiae. By the second step, the transformation space is estimated in terms of a clustering method, and multi-resolution technique ${ }^{[9]}$ is employed to obtain an optimal transformation model. Finally, the global similarity is calculated by accumulating all local similarity by using plus rule. Experimental results show that our method is promising in an AFIS.

\section{Fingerprint enhancement based on DSS}

In computer vision, scale-space theory is often used for image segmentation and edge extraction. Those are considered as the object's outline. Fingerprint image has its own characteristics, such as the flow-like pattern, which means ridges are alternated with valleys. In our algorithm, a fingerprint is first decomposed into a series of images to reduce noises in different scales. All the enhanced images are combined to get a more credible image. Each time we reduce the noise to some extent, and with several iterations, the final enhanced image was obtained. 


\subsection{Linear scale space}

Scale-space theory provides a canonical framework for modeling visual operation at multiple scales. The scale-space representation $L: R^{2} \otimes R \rightarrow R^{2}$ of a two-dimensional image $f$ is defined as one-parameter family of functions obtained by convolving $f$ with Gaussian kernel:

$$
\left\{\begin{array}{l}
L(x, y ; t)=f(x, y) \otimes g_{t}(x, y, \theta) \\
g_{t}(x, y, \theta)=\frac{2}{2 \pi t^{2}} e^{-\frac{\left((x \cos \theta)^{2}+(y \sin \theta)^{2}\right)}{2 t^{2}}}
\end{array}\right.
$$

where the parameter $t$ is called scale parameter and $\theta$ is the direction of the pixel $(x, y)$ in $f$. Although fingerprints are non-isotropic, we use the linear scale-space to detect the feature because the ridge is a line in local region. And the convolution is along the ridge direction, as the filter is adapted to ridge direction. Equivalently, these family images can be generated by the solution to the diffusion equation:

$$
\partial_{t} L=\frac{1}{2} \nabla^{T} \nabla L=\frac{1}{2}\left(\partial_{x^{2}}+\partial_{y^{2}}\right) L
$$

with initial condition $L(x, y ; 0)=f(x, y)$.

In scale-space, with the increase of the scale, the number of the details decreases and the noise is successively suppressed gradually. The feature of the image in large scale exists in the image of small scale as well, while the weak signal will disappear in the large scale space. Those features existing in the image of large scale are global structure. Using a series of scales $\sigma_{1}, \sigma_{2}, \ldots, \sigma_{n 0}$ to filter the image $f$, the scale-spaces are denoted as $L_{\sigma_{k}}\left(n_{0} \geqslant k \geqslant 1\right)$ with scale $\sigma_{k}$. We define the details between two scales as

$$
D_{k}=L_{\sigma_{k}}-L_{\sigma_{k+1}} ; \quad\left(n_{0}-1\right) \geqslant k \geqslant 1 \text {. }
$$

Let $D_{0}=f-L_{\sigma_{1}}$, we obtain the scale-space representation and its details, and construct them to enhance the image $f$ :

$$
f=L_{\sigma_{n}}+\sum_{k=0}^{n-1} D_{k} .
$$

\subsection{Pre-processing with DSS}

There are two problems in fingerprint enhancement by using scale-space theory. One is how to select different scales, and the other is how long the series is. We discuss the problem as follows.

Analogical to the wavelet, we use the dyadic scale to decompose a fingerprint. The scale is selected as $\sigma_{k}=2^{k}\left(n_{0} \geqslant k \geqslant 1\right)$, and it is called dyadic, the scale-space is known 
as DSS. The length of the series is decided by $n_{0}$. We define the mean width of fingerprint as $w$, calculated with Lin et al.'s method ${ }^{[1]}$, and $n_{0}=\left\lceil\log _{2}(2 w)\right\rceil$. We get the smoothed image of the fingerprint image $f$ by using DSS $L_{\sigma_{k}}\left(n_{0} \geqslant k \geqslant 1\right), L_{\sigma_{k}}=f \otimes g_{2^{k}}$. With formulas (3) and (4), the detail image, $D_{k}\left(n_{0}-1 \geqslant k \geqslant 0\right)$, is obtained. The effect of the noise is reduced in the image $L_{\sigma_{k}}$. The gray values reflect the ridge value. And $D_{k}$ contains the information, so does $L_{\sigma_{k}}$, but at the stage of $L_{\sigma_{k+1}}$ the information is lost. Because of the noise in $D_{k}$, we smooth it with scale $2^{k+1}$, that is, $L_{\sigma_{k}}^{\prime}=D_{k} \times g_{2^{k+1}}$.

Let $E_{k-1}$ be the image obtained through the scale $2^{k-1}$. The image $E_{k}$ at the scale $2^{k}$ is calculated with formulas (5) and (6):

$$
E_{k}(i, j)=\left\{\begin{array}{lr}
0, & t \neq 0, E_{k-1}(i, j) \leqslant\left|L_{\sigma_{k+1}}(i, j)-L_{\sigma_{k}}^{\prime}(i, j)\right| ; \\
255, & t=0 \text { or } E_{k-1}(i, j) \geqslant\left(L_{\sigma_{k+1}}(i, j)+L_{\sigma_{k}}^{\prime}(i, j)\right) ;(5) \\
\frac{255}{t}\left(E_{k-1}(i, j)-\left|L_{\sigma_{k+1}}(i, j)-L_{\sigma_{k}}^{\prime}(i, j)\right|\right), t \neq 0, E_{k-1}(i, j) \leqslant\left(L_{\sigma_{k+1}}(i, j)+L_{\sigma_{k}}^{\prime}(i, j)\right)
\end{array}\right.
$$

where

$$
t=\left\{\begin{array}{l}
L_{\sigma_{k+1}}(i, j)+L_{\sigma_{k}}^{\prime}(i, j)-E_{k-1}(i, j),\left(L_{\sigma_{k+1}}(i, j)+L_{\sigma_{k}}^{\prime}(i, j)\right) \leqslant 255 \\
255-\left|L_{\sigma_{k+1}}(i, j)-L_{\sigma_{k}}^{\prime}(i, j)\right|, \text { otherwise. }
\end{array}\right.
$$

Because the gray value in the $L_{\sigma_{k+1}}$ is smoothed to reduce the noise, the gray value $L_{\sigma_{k}}^{\prime}$ is credible. Then we compare the gray value of $L_{\sigma_{k+1}}$ with $L_{\sigma_{k}}^{\prime}$ and get the result image which is affected by noises. The noise is reduced through this step time by time.

Since a fingerprint may be corrupted by noise during capture because of impression, skin, reader, etc., Luo et al.'s knowledge-based fingerprint enhancement method $^{[10]}$ is used to reduce the effects. And Farina's minutia extraction method ${ }^{[11]}$ is applied to detect minutiae in the thinned fingerprint.

\section{Minutiae matching using clustering method}

In our matching, fingerprint $F$ is matched to fingerprint $G$ with the following steps. We first introduce minutia-simplex, a relative structure among minutiae, for its good trade-off between performance and memory expense. Then we roughly match minutiasimplex, calculate the local similarity, and estimate the transformation model and its corresponding space in terms of clustering. Finally, we search for an optimal transformation model using multi-resolution technique to obtain the maximal mutual information between two fingerprints.

3.1 Feature analysis with clustering method

A fingerprint feature is produced with weight $p_{i}\left(1 \geqslant p_{i} \geqslant 0\right)$ for it is interrupted by 
noises or bad quality. In our study, we estimate the distribution of these features in terms of $k$-means clustering method ${ }^{[12]}$.

Let $X=\left\{X_{i}=\left(x_{i}^{(1)}, \ldots, x_{i}^{(N)}\right)^{T} ;|X| \geqslant i \geqslant 1\right\}$ denote a feature set. Assume that all features in $X$ are independent, and the elements of each feature are also independent. Then analyze the distribution of all features $X_{i} \in X$ with the following steps:

Step 1. Set $X$ is represented with $X^{(k)}=\left\{x_{i}^{(k)} ;|X| \geqslant i \geqslant 1, N \geqslant k \geqslant 1\right\}$ and $X=\left\{X^{(1)}, \ldots\right.$, $\left.X^{(N)}\right\}$. Let $x^{(k)}$ denote the $k$ th element of a feature. To build the histogram of $x^{(k)}, H\left(x^{(k)}\right)$ $(N \geqslant i \geqslant 1)$ with formula (7). $H\left(x^{(k)}\right)$ represents the distribution of $x^{(k)}$.

$$
H\left(x^{(k)}\right)=\frac{\sum_{\left\{j ; x_{j}^{(k)}=x^{(k)}\right\}} p_{j}}{\Sigma p_{j}} .
$$

Step 2. To filter $H\left(x^{(k)}\right)$ with formula (8) to reduce the effects of noises on the distribution of $x^{k}$.

$$
f(x)=\frac{(1-\cos (x \pi / d))}{2} ; 1 \leqslant x \leqslant 2 d+1
$$

Step 3. To calculate the confidence interval of $x^{(k)}$ under a confidence. Theoretically, $x^{(k)}$ most probably happens where $H\left(x^{(k)}\right)$ is maximal, that is, $H\left(u_{k}\right)=\max \left\{H\left(x^{k}\right)\right\}$. However, $u_{k}$ is affected by noises so that we use a confidence interval $U_{k}=\left[u_{k}-\lambda_{k} \sigma_{k}, u_{k}+\lambda_{k} \sigma_{k}\right]$ instead of a point where $u_{k}$ and $\sigma_{k}$ are the mean and standard deviation of $x^{(k)}$ respectively. And $\lambda_{k}\left(\lambda_{k}>0\right)$ is an empirical threshold. There is a probability that $x^{(k)}$ will happen in the interval $U_{k}$ is $2 \phi\left(\lambda_{k}\right)-1$.

It is difficult to obtain the variance $u_{k}$ and $\sigma_{k}$. Therefore, we indirectly estimate them by the following steps. We first find the maximum of $H\left(x^{(k)}\right)$ to obtain $u_{k}$, that is, to find a site $x^{(k)}=u_{k} \pm \lambda_{k} \sigma_{k}$ where $H\left(x^{(k)}\right)=\exp \left(-\frac{\lambda_{k}^{2}}{2}\right) \times \max \left\{H\left(x^{(k)}\right)\right\}$. Then we estimate the confident interval $U_{k}$ with the confidence $2 \phi\left(\lambda_{k}\right)-1$. We align $\lambda_{k}$ according to confidence and its confident interval. If $\lambda_{k}=\sqrt{2 \ln 2}=1.38, \exp \left(-\frac{\lambda_{k}^{2}}{2}\right)=0.5$, its corresponding confidence and confidence interval are 0.8328 and $U_{k}=\left[u_{k}-1.38 \sigma_{k}, u_{k}+1.38 \sigma_{k}\right]$.

Step 4. To calculate the confidence interval of feature $X_{i}$. For the confidence interval of all $x^{(k)}$, and their corresponding confidence interval $U_{k}$, the confidence interval of feature $X_{i}$ is $S=\left\{x^{(k)} \in U_{k} ; N \geqslant k \geqslant 1\right\}\left(\lambda_{1}=\lambda_{2}=\ldots=\lambda_{N}=\lambda\right)$. If a feature is outside $S$, it is unimportant. If an estimated space with $\lambda$ is far greater than a preset space, the feature set $X$ is considered as a disorder set. 


\subsection{Fingerprint representation in our method}

A comprehensive minutia includes its minutiae and its local associated ridge information. Let $M^{F}=\left\{M_{i}^{F}=\left(x_{i}^{F}, y_{i}^{F}, \alpha_{i}^{F}, \quad \beta_{i}^{F}, l_{i j}^{F}, \quad \varphi_{i j}^{F}\right)^{T} ;\left|M^{F}\right| \geqslant i \geqslant 1, L \geqslant j \geqslant 1\right\}$ denote the comprehensive minutiae set of fingerprint $F$. A comprehensive minutia $M^{F}{ }_{i}$ is represented by a feature vector $\left(x_{i}^{F}, y_{i}^{F}, \alpha_{i}^{F}, \beta_{i}^{F}, l_{i j}^{F}, \varphi_{i j}^{F}\right)^{T}\left(\left|M^{F}\right| \geqslant i \geqslant 1, L \geqslant j \geqslant 1\right)$, where 1) $x_{i}^{F}$ and $y_{i}^{F}$ are the coordinates; 2) $\alpha_{i}^{F}$ is the tangent direction at $M_{i}^{F}$;3) $\beta_{i}^{F}$ is the local grey variance of a $16 \times 16$ area whose centre is $M_{i}^{F}$. We replace the type of the minutia with its local grey variation since the type of $M_{i}^{F}$, ending or bifurcating, is corrupted by noises; 4) $\varphi_{i j}^{F}$ denotes the direction from $M_{i}^{F}$ to the $j$ th sampled point on the ridge beginning at $M_{i}^{F}$, and $l_{i j}^{F}$ denotes the distance from $M_{i}^{F}$ to the $j$ th sampled point. All $\left(l_{i j}^{F}, \varphi_{i j}^{F}\right)$ represent the ridge information associated with the minutiae; 5) $\left|M^{F}\right|$ denotes the number of minutiae in fingerprint $F$ and $L$ the number of sampled points on a ridge.

A fingerprint is represented by relative structures among minutiae. Let $E^{F}=\left\{E_{i}^{F} ;\left|E^{F}\right|\right.$ $\geqslant i \geqslant 1\}$ denote all relative structures, where $\left|E^{F}\right|$ is the size of set $E^{F}$. Features of $E_{i}^{F}$ can be decomposed into transformation-invariant feature vector $C_{i}^{F}$ and transformation variant feature vector $V_{i}^{F}$ according to their relativity with transformation. Let $C^{F}=\left\{C_{i}^{F}\right\}, V^{F}=\left\{V_{i}^{F}\right\}$, and $E_{i}^{F}=\left\{C_{i}^{F}, V_{i}^{F}\right\}$. For an optimal transformation $T$, it is possible to obtain the maximal mutual information between fingerprints $F$ and $G$, that is, the maximal mutual information between all $V^{F}$ and $V^{G}$. Their relation is described by $T \propto\left(V^{F}-V^{G}\right) \Rightarrow T=H\left(V^{F}-V^{G}\right), H(\cdot)$ is the function of $V^{F}-V^{G}$.

In our study, a minutia-simplex is employed to describe a 2nd-order relative structure among minutiae. Compared with minutia-triplet ${ }^{[3]}$, minutia-simplex has good trade-off between performance and expense. Minutia-simplex is built as follows.

For each pair of minutiae $M_{i}^{F}$ and $M_{j}^{F}\left(\left|M^{F}\right| \geqslant i, j \geqslant 1\right)$ in $M^{F}$, if their distance $d\left(M_{i}^{F}, M_{j}^{F}\right)=\sqrt{\left(x_{i}^{F}-x_{j}^{F}\right)^{2}+\left(y_{i}^{F}-y_{j}^{F}\right)^{2}}$ satisfies $d_{l} \leqslant d\left(M_{i}^{F}, \quad M_{j}^{F}\right) \leqslant d_{h}, \quad M_{i}^{F}$ and $M_{j}^{F}$ are connected as a minutia-simplex described by a feature vector composed of index features $\left(r_{k}^{F}, e_{k}^{F}\right)$, transformation-invariants $\left(l_{k}^{F}, \beta_{k 1}^{F}, \beta_{k 2}^{F}, u_{k}^{F}, v_{k}^{F}\right)$, and transformation-variants $\left(\theta_{k}^{F}, \quad \varphi_{k 1 t}^{F}, \quad \varphi_{k 2 t}^{F}, \quad \alpha_{k 1}^{F}, \quad \alpha_{k 2}^{F}\right)(L \geqslant t \geqslant 1)$ where

1) Index features $r_{k}^{F}$ and $e_{k}^{F}$ denote the serial numbers of two minutiae associated 
with $E_{k}^{F}$, i.e. $r_{k}^{F}=i$ and $e_{k}^{F}=j$.

2) $l_{k}^{F}, \beta_{k 1}^{F}, \beta_{k 2}^{F}, u_{k}^{F}$ and $v_{k}^{F}$ describe the transformation-invariant relative features of $E_{k}^{F}$. They are irrelevant with rotation and translation, where $l_{k}^{F}=d\left(M_{i}^{F}, M_{j}^{F}\right)$, $\beta_{k 1}^{F}=\beta_{i}^{F}, \quad \beta_{k 2}^{F}=\beta_{j}^{F}, u_{k}^{F}=\alpha_{i}^{F}-\theta_{k}^{F}, \quad v_{k}^{F}=\alpha_{j}^{F}-\theta_{k}^{F}$.

3) $\theta_{k}^{F}, \varphi_{k 1 t}^{F}, \varphi_{k 2 t}^{F}, \quad \alpha_{k 1}^{F}$ and $\alpha_{k 2}^{F}(L \geqslant t \geqslant 1)$ represent the transformation-variant relative features of $E_{k}^{F}$ where $\theta_{k}^{F}=\arctan \left(\begin{array}{c}y_{i}^{F}-y_{j}^{F} \\ x_{i}^{F}-x_{j}^{F}\end{array}\right) ; \quad \varphi_{k 1 t}^{F}=\varphi_{i t}^{F}$ and $\varphi_{k 2 t}^{F}=\varphi_{j t}^{F}(L$ $\geqslant t \geqslant 1)$.

Let $E^{F}=\left\{E_{k}^{F} ;\left|E^{F}\right| \geqslant k \geqslant 1\right\}$ denote all simplexes in fingerprint $F$ where $\left|E^{F}\right|$ is the size of set $E^{F} . E^{F}$ represents the global characteristics of fingerprint more accurately than the minutia set $M^{F} \cdot\left|E^{F}\right|$ is much smaller than $\left(\begin{array}{c}\left|M^{F}\right| \\ 2\end{array}\right)$ because many minutia pairs do not satisfy $d_{l} \leqslant d\left(M_{i}, M_{j}\right) \leqslant d_{h} . d_{l}$ and $d_{h}$ are two empirical thresholds dependent on sensors.

Let $d V=\left\{d V_{k}=\left(d \theta_{k}, d x_{k}, d y_{k}\right)^{T}\right\}$ denote the bias set of all transformation-variant relative features of minutia-simplexes, where $d V_{k}$ is a vector describing biases of transformation-variants of the $k$ th minutia-simplex with formula (9).

$$
\left\{\begin{array}{c}
d x_{k}=\frac{\sum_{i=1}^{2} d x_{k i}}{2}, \quad d y_{k}=\frac{\sum_{i=1}^{2} d y_{k i}}{2}, \\
d \theta_{k}=\frac{\left(d \theta_{k}+\sum_{i=1}^{2} d \alpha_{k i}+\sum_{i=1}^{L} d \varphi_{k 1 i}+\sum_{i=1}^{L} d \varphi_{k 2 i}\right)}{2 L+3} .
\end{array}\right.
$$

\subsection{Transformation space estimation}

In our study, affine transformation is employed to model $H(\bullet)$ for its good matching performance. Compared with Feng et al.'s ${ }^{[13]}$ method, $k$-clustering method is employed in our work to estimate the transformation space. Let $H(\bullet)$ denote all transformation models in fingerprint matching. $H\left({ }^{\bullet}\right)$ is understood as the function of $d V=V^{F}-V^{G}$, that is, $H(\bullet)=\left\{H_{d}(\bullet), H_{x}(\bullet), H_{y}(\bullet)\right\}$. In our study, matching is performed in the polar system and center points of fingerprints $F$ and $G$ are calculated in advance. One of the most challenging tasks in matching is to estimate an optimal interval of rotation transformation parameter with the following steps: 
Step 1. To calculate $d V$. For an input minutia-simplex $E_{m}^{F}\left(\left|E^{F}\right| \geqslant m \geqslant 1\right)$ and its corresponding template $E_{n}^{G}\left(\left|E^{G}\right| \geqslant n \geqslant 1\right)$, their transformation-variant feature biases $d V_{k}$ are calculated with formula (9).

Step 2. To test all elements in $d V . d V_{k}$ is invalid if $C_{m}^{F}$ is not equal to $C_{n}^{G}$. Considering the noise in matching, $C_{m}^{F}$ is matched to $C_{n}^{G}$ in terms of tolerance deviation, and their similarity is denoted with $S\left(C_{m}^{F}, C_{n}^{G}\right)$ with formula (10). $S\left(C_{m}^{F}, C_{n}^{G}\right)$ is also seen as the weight of $d V_{k}$. In formula (10), $\varepsilon$ is a threshold indicating the tolerance deviation of transformation-variant relative features.

$$
S\left(C_{m}^{F}, C_{n}^{G}\right)=\left\{\begin{array}{l}
0,\left\|C_{m}^{F}-C_{n}^{G}\right\| \geqslant \varepsilon ; \\
1-\frac{\left\|C_{m}^{F}-C_{n}^{G}\right\|}{\varepsilon}, \text { otherwise. }
\end{array}\right.
$$

Step 3. To estimate rotation-transformation interval. For $d V_{k}$ and its corresponding $S\left(C_{m}^{F}, C_{n}^{G}\right)$, the distribution $H_{\theta}(\bullet)$ and confidence interval of transformation $\theta$ can be estimated with the clustering method described above.

Step 4. To calculate the initial rotation transformation model. For the transformation space $S$ and the distribution of rotation transformation parameter $H_{\theta}(\bullet)$, an initial transformation model $\theta_{0}$ is calculated with formula (11) in terms of geometric means. $u_{\theta}$ and $\sigma_{\theta}$ can be calculated with the scheme described above when $\lambda_{\theta}=1.38$.

$$
\theta_{0}=\frac{\sum_{u_{\theta}-\lambda_{\theta} \sigma_{\theta}}^{u_{\theta}+\lambda_{\theta} \sigma_{\theta}} \theta_{i} H_{\theta}\left(\theta_{i}\right)}{\sum_{u_{\theta}-\lambda_{\theta} \sigma_{\theta}}^{u_{\theta}+\lambda_{\theta} \sigma_{\theta}} H\left(\theta_{i}\right)} .
$$

To evaluate the performance of the initial rotation-parameter, an experiment was performed over 20 fingerprints randomly selected from the fingerprint databases of FVC2002 (the 2nd International Fingerprint Verification Competition in $2002^{[14]}$ ). For fingerprint $G$, its transformed fingerprint $G^{\prime}$ was produced by rotating $F$ with an angle $\theta$ $\left(15^{\circ} \geqslant \theta \geqslant-15^{\circ}\right)$, with reference to its central point. And rotation parameters $\theta^{\prime}$ between $G$ and $G^{\prime}$ were estimated from their $H_{\theta}(\bullet)$. The result is shown in Fig. 1. The mean of the absolute errors $\left|\theta-\theta^{\prime}\right|$ was $0.3318^{\circ}$.

To evaluate the performance of $H_{\theta}(\bullet)$, a pair of fingerprints were selected from the same finger, as shown in Fig. 2(a), while two other pairs of fingerprints were selected from two different fingers respectively, see Fig. 2(b) and (c). The two fingerprints of 


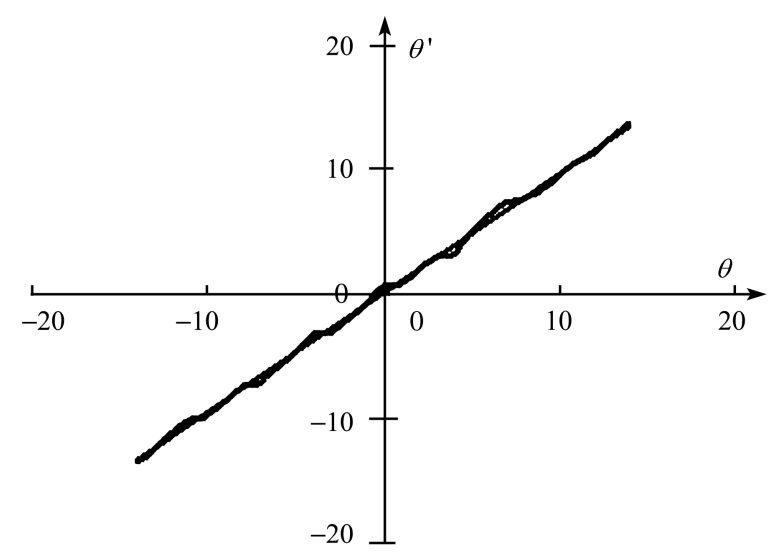

Fig. 1. Performance of estimating initial rotation-parameter.
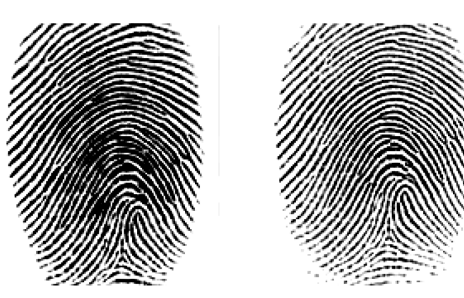

(a)

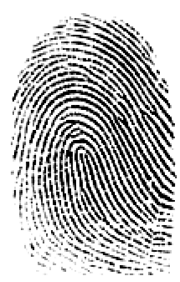

(b)
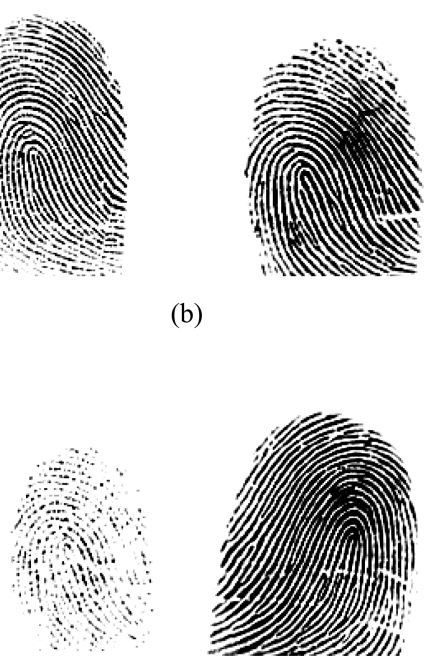

(c)

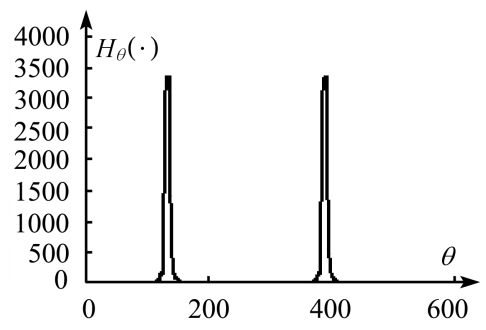

(a)'

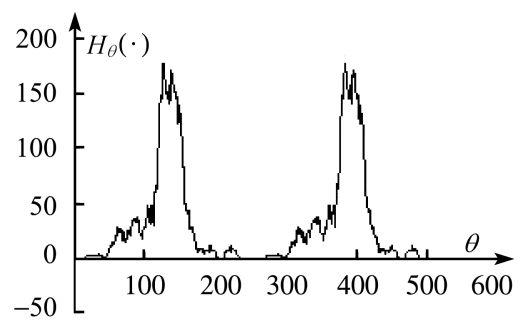

(b)'

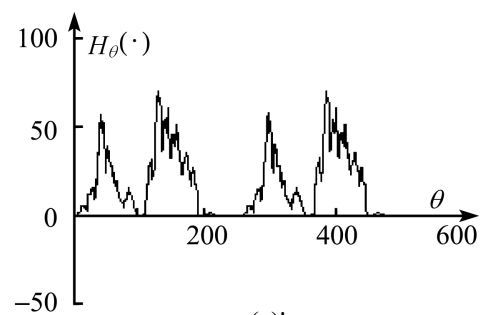

(c)'

Fig. 2. Performance of $H_{\theta}(\bullet)$ s of three pairs of fingerprints. (a) shows two fingerprints from the same finger while (b) and (c) display two pairs of fingerprints from two different fingers. 
the second pair are similar. Fig. 2(a)', (b)' and (c)' show that the $H_{\theta}(\bullet$ ) of the first pair is of normal distribution while two other $H_{\theta}(\bullet)$ s are of random distribution caused by mismatches produced by spurs or deformation although the second $H_{\theta}(\bullet)$ is more regular than that of the third one.

\subsection{Fingerprint matching}

Fingerprint matching is to find the maximal mutual information between input and template features with an optimal transformation model. Our matching method consists of the following steps:

Step 1. To estimate the transformation space in matching two fingerprints $F$ and $G$ described in the previous section and to get an initial transformation model $T_{0}$ with formula (11).

Step 2. To calculate the similarity between minutia pair $M^{F}{ }_{i}$ and $M_{j}^{G}$ with formula (12) where $\Omega_{m}^{F}=\left\{E_{m}^{F} ;\left(r_{m}^{F}=i\right)\right.$ or $\left.\left(e_{m}^{F}=i\right)\right\}$ and $\Omega_{j}^{G}=\left\{E_{n}^{G} ;\left(r_{n}^{G}=j\right)\right.$ or $\left.\left(e_{n}^{G}=j\right)\right\}$.

$$
S\left(M_{i}^{F}, M_{j}^{G}\right)=\sum_{E_{n}^{F} \in \Omega_{i}^{F}, E_{m}^{G} \in \Omega_{j}^{G}} S\left(C_{m}^{F}, C_{n}^{G}\right) .
$$

All the similarity of minutia pairs is sorted in descending order of value. We select the first $K$ minutia pairs as the center point candidates of fingerprints $F$ and $G$ respectively. Let $\left\{\left(\left(x_{k 0}^{F}, y_{k 0}^{F}\right),\left(x_{k 0}^{G}, y_{k 0}^{G}\right)\right) ; K \geqslant k \geqslant 1\right\}$ denote the set of these minutia pairs.

Step 3. For initial transformation model $T_{0}$ and the center point candidate set, to calculate the global similarity between fingerprints $F$ and $G$.

1) To obtain a transformation model $T_{i}$ using multi-resolution technique in space $S$.

2) To select a center point pair $\left(x_{k 0}^{F}, y_{k 0}^{F}\right)$ and $\left(x_{k 0}^{G}, y_{k 0}^{G}\right)(K \geqslant k \geqslant 1)$ as the center points of fingerprints $F$ and $G$ respectively.

3) To transform all minutiae in $M^{F}$ and $M^{G}$ into the responding polar systems with the transformation model $T_{i}$ with reference to their center points respectively.

4) To validate $S\left(C_{m}^{F}, C_{n}^{G}\right)$. If the two aligned minutiae associated with $E_{m}^{F}$ are located within the bounded boxes ${ }^{[15]}$ centred at the two aligned minutiae associated with $E_{n}^{G}$ respectively, minutiae-simplexes $E_{m}^{F}$ and $E_{n}^{G}$ are assumed to be similar, then, $S\left(C_{m}^{F}, C_{n}^{G}\right)$ is valid. Otherwise, they are considered as invalid and $S\left(C_{m}^{F}, C_{n}^{G}\right)$ is deleted from the matching result set.

5) To estimate the similarity between transformation-variants of $E_{m}^{F}$ and $E_{n}^{G}$ by matching the aligned transformation-variant relative features of $E_{m}^{F}$ and $E_{n}^{G}$, which 
means vector $\left(\theta_{m}^{F}+\theta_{i}, \quad \varphi_{m 1 p}^{F}+\theta_{i}, \quad \varphi_{m 2 p}^{F}+\theta_{i}, \quad \alpha_{m 1}^{F}+\theta_{i}, \quad \alpha_{m 2}^{F}+\theta_{i}\right)$ is matched to vector $\left(\theta_{n}^{G}, \varphi_{n 1 p}^{G}, \varphi_{n 2 p}^{G}, \alpha_{n 1}^{G}, \alpha_{n 2}^{G}\right)$ where $\theta_{i}$ is the rotation parameter of $T_{i}$. Let $S_{(i k)}\left(V_{m}^{F}\right.$, $V_{n}^{G}$ ) denote the similarity between the two vectors calculated with formula (10), all local matching results $S\left(C_{m}^{F}, C_{n}^{G}\right)$ are tested and two new similarity sets $S_{(i k)}\left(C_{m}^{F}, C_{n}^{G}\right)$ and $S_{(i k)}\left(V_{m}^{F}, V_{n}^{G}\right)$ are produced.

6) To calculate the global similarity between two fingerprints $F$ and $G$ in terms of plus rule with formulas (13) and (14) with transformation model $T_{i}$ and centered point pair $\left(\left(x_{k 0}^{F}, y_{k 0}^{F}\right),\left(x_{k 0}^{G}, y_{k 0}^{G}\right)\right)$. In formula (13), $n_{s}, n_{l}, e_{s}$, and $e_{l}$ are empirical thresholds.

$$
\begin{aligned}
S_{(i k)}= & f_{G L M}\left(\left|S_{(i k)}(F, G)\right| ; n_{s}, n_{l}\right) \\
& \times f_{G L M}\left(\sum\left(S_{(i k)}\left(V_{m}^{F}, V_{n}^{G}\right)+S_{(i k)}\left(C_{m}^{F}, C_{n}^{G}\right)\right) ; e_{s}, e_{l}\right), \\
& f_{G L M}\left(x ; \delta_{s}, \delta_{l}\right)= \begin{cases}0, & x<\delta_{s}, \\
\frac{x-\delta_{s}}{\delta_{l}-\delta_{s}}, & \delta_{s} \leqslant x<\delta_{l}, \\
1, & x \geqslant \delta_{l} .\end{cases}
\end{aligned}
$$

7) If $S_{(i k)}(F, G) \geqslant \rho$, the matching is over; otherwise, go to step 3-(2). The matching is looped $K$ times until every center point pair in $\left\{\left(\left(x_{k 0}^{F}, y_{k 0}^{F}\right),\left(x_{k 0}^{G}, y_{k 0}^{G}\right)\right) ; K \geqslant k \geqslant 1\right\}$ is tested.

8) To continue step 3-(1) to find a new transformation model $T_{i+1}$ from the space $S$ by using multi-resolution technique. If the matching is looped more than $P$ times, the matching is over. And $\max \left\{S_{(i k)}\right\}$ is thought as the final global similarity between fingerprints $F$ and $G$.

\section{Results}

Experiments were performed over the fingerprint databases of FVC2002 ${ }^{[14]}$ instead of data sets of the US National Institute of Standards and Technology (NIST) ${ }^{[16-18]}$ for these fingerprint databases of the 1st International Fingerprint Verification Competition in 2000 (FVC2000 $^{[19]}$ ) and FVC2002 are more suitable for evaluating a matching method in application ${ }^{[19]}$. And our method was evaluated by FVC2002 and it ranked the seventh.

\subsection{Results of enhancement}

The performance of the enhancement algorithm was evaluated over the four fingerprint databases of FVC2002. Fig. 3 illustrates four typical examples of the enhancement performance. In Fig. 3, (a-1), (b-1), (c-1) and (d-1) are the 23rd fingerprints of the four 


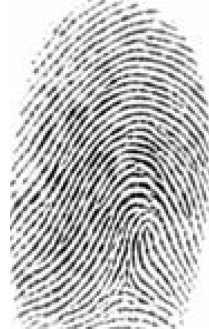

(a-1)

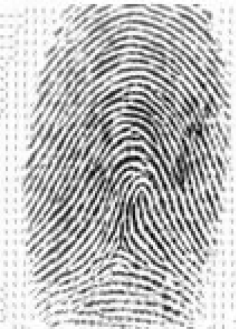

(a-2)

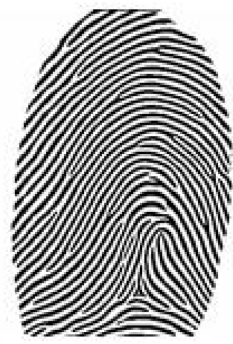

(a-3)

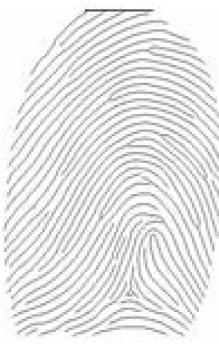

(a-4)

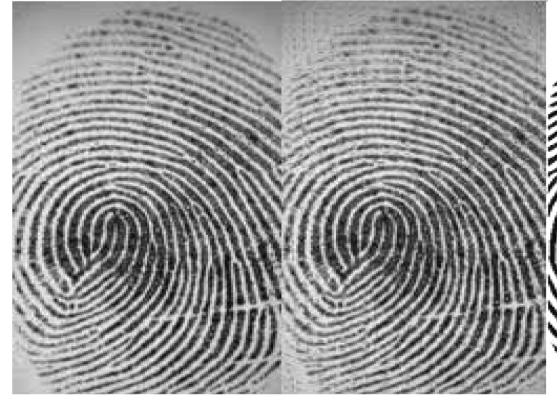

(b-1)

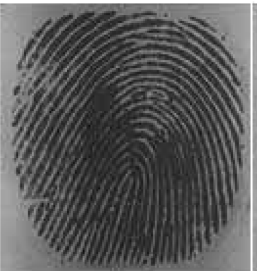

(c-1)

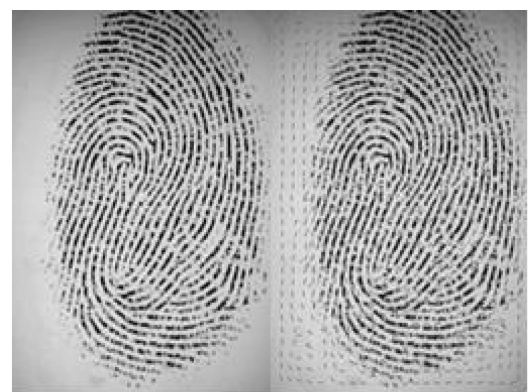

$(\mathrm{d}-1)$ (b-2)

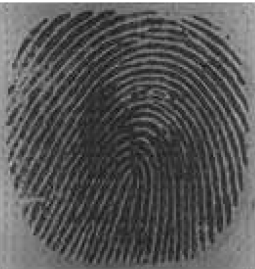

(c-2)

(d-2)

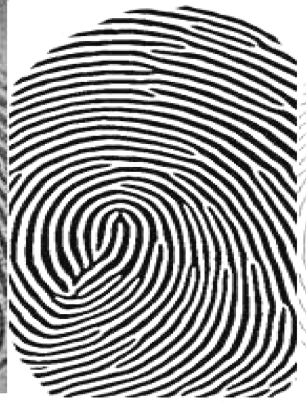

(b-3)

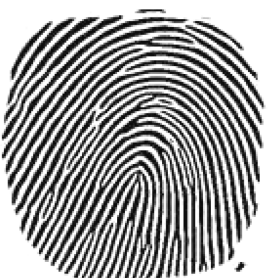

(c-3)

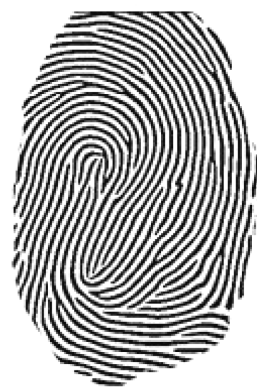

(d-3)

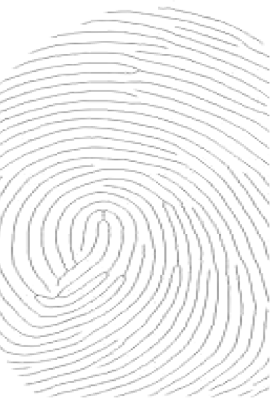

(b-4)

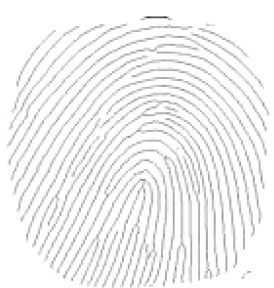

(c-4)

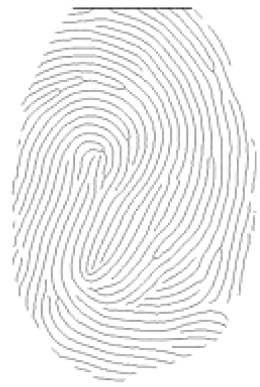

(d-4)

Fig. 3. Performance of our enhancement method based on DSS over the fingerprints of FVC2002.

fingerprint databases of FVC2000 respectively; (a-2), (b-2), (c-2) and (d-2) represent their orientation field images; (a-3), (b-3), (c-3) and (d-3) indicate their binary images; and (a-4), (b-4), (c-4) and (d-4) show their thinned images. The average time of en- 
hancement are $0.41,0.34,0.91$ and 0.26 s respectively; experiments on enhancement were conducted on a Pentium III450 MHz machine by using windows 2000. The results confirm that the enhancement method outperforms other methods ${ }^{[1]}$.

\subsection{Results of matching}

Our matching performance was evaluated over the four fingerprint databases of FVC2002 as shown in Fig. 4 and Table 1, which were provided by FVC2002.

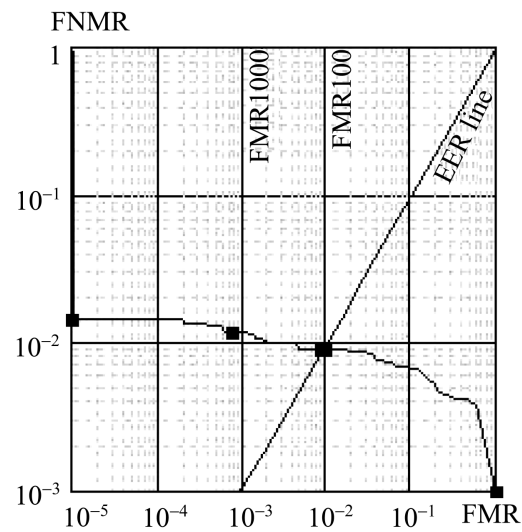

(a)

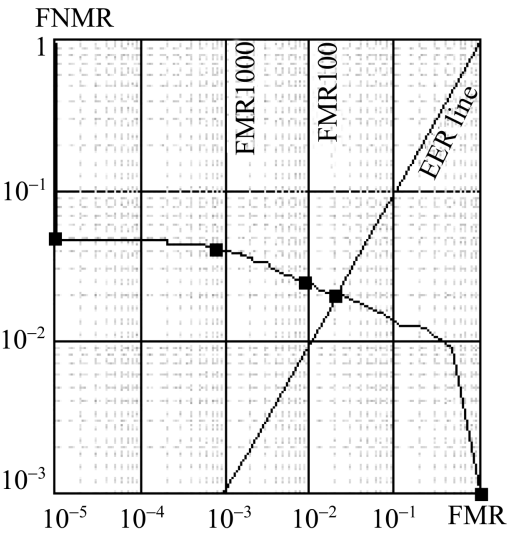

(c)

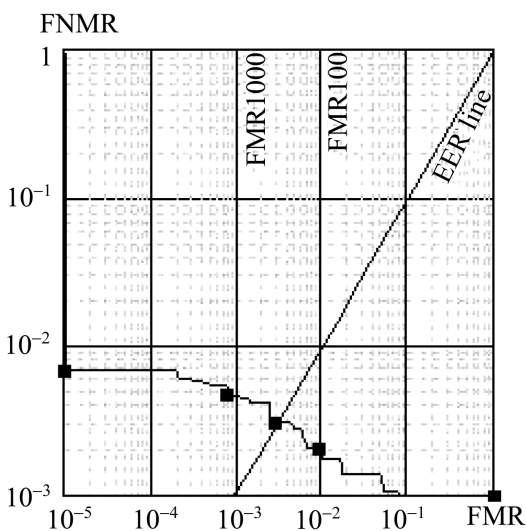

(b)

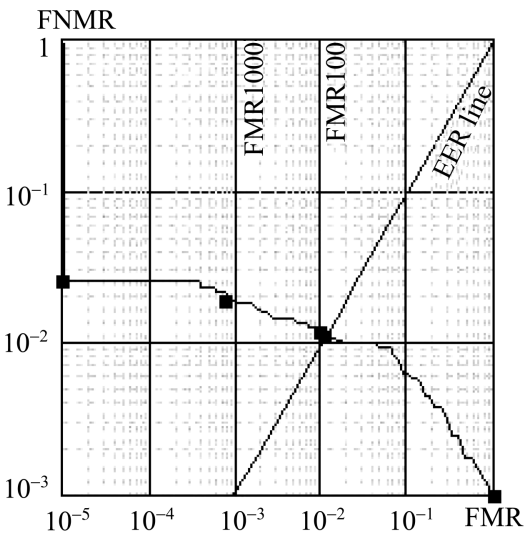

(d)

Fig. 4. ROCs of our matching method over the four fingerprint databases of FVC2002, provided by FVC2002.

Table 1 Performance indices of the four fingerprint databases of FVC2002, provided by FVC2002

\begin{tabular}{ccccccccc}
\hline Database & $\begin{array}{c}\text { EER } \\
(\%)\end{array}$ & $\begin{array}{c}\text { EER* } \\
(\%)\end{array}$ & $\begin{array}{c}\text { FMR100 } \\
(\%)\end{array}$ & $\begin{array}{c}\text { FMR1000 } \\
(\%)\end{array}$ & $\begin{array}{c}\text { ZeroFMR } \\
(\%)\end{array}$ & $\begin{array}{c}\text { ZeroFNMR } \\
(\%)\end{array}$ & $\begin{array}{c}\text { AVG } \\
\text { enroll } \\
\text { time (s) }\end{array}$ & $\begin{array}{c}\text { AVG } \\
\text { match } \\
\text { time (s) }\end{array}$ \\
\hline DB1_a & 0.91 & 0.91 & 0.93 & 1.21 & 1.50 & 100.00 & 0.35 & 0.37 \\
DB2_a & 0.30 & 0.30 & 0.21 & 0.50 & 0.71 & 100.00 & 0.50 & 0.52 \\
DB3_a & 2.05 & 2.05 & 2.54 & 4.25 & 5.04 & 100.00 & 0.27 & 0.27 \\
DB4_a & 1.14 & 1.14 & 1.21 & 1.96 & 2.64 & 100.00 & 0.28 & 0.29 \\
\hline
\end{tabular}




\section{Conclusion and discussion}

This paper introduces a matching algorithm by clustering similarity with the novel points: DSS enhancement and transformation space estimation in terms of clustering method. The outstanding performance of this method confirms it is promising in an AFIS.

However, this method is sensitive to the bad-quality fingerprints. They affect the reliability of minutiae, rotation parameter, center points, and therefore affect matching performance. Further investigation will be carried out to improve our method by minimizing false match which occasionally occurs under the condition of large deformation and very poor-quality fingerprints ${ }^{[20]}$. 1) Global pattern and features and a hybrid matching technique will be inspected to reduce the sensitivity of poor quality fingerprint for it is hard for minutia-based matching method ${ }^{[21]}$ and therefore improve the performance of the proposed method over these bad quality fingerprints. 2) The technique that employs a multi-resolution search strategy to calculate the optimal transformation is studied as well.

Acknowledgements This work was supported by the Project of National Science Fund for Distinguished Young Scholars of China (Grant No. 60225008), the National Natural Science Foundation of China (Grant No. 60332010), and the Project for Young Scientists' Fund of National Natural Science Foundation of China (Grant No. 60303022).

\section{References}

1. Lin, H., Wan, Y. F., Jain, A. K., Fingerprint image enhancement: algorithm and performance evaluation, IEEE Transactions on Pattern Analysis and Machine Intelligence, 1998, 20(8): 777-789.

2. Gold, S., Rangarajan, A., A graduated assignment algorithm for graph matching, IEEE Transaction on Pattern Analysis and Machine Intelligence, 1996, 18(4): 377-388.

3. Jiang, X. D., Yau, W. Y., Fingerprint minutiae matching based on the local and global structures, in Proceedings of the 15th International Conference on Pattern Recognition (eds. Sanfeliu, A. et al.), Bercelona: IEEE CS-Press, 2000, 2: 1042-1045.

4. Jain, A. K., Prabhakar, S., Lin, H. et al., Filterbank-based fingerprint matching, IEEE Transaction on Image Processing, 2000, 9(5): 846-859.

5. Sujan, V. A., Mulqueen, M. P., Fingerprint identification using space invariant transforms, Pattern Recognition Letters, 2002, 23(5): 609-619.

6. Luo, X. P., Tian, J., A minutia matching algorithm in fingerprint verification, in Proceedings of the 15th International Conference on Pattern Recognition (eds. Sanfeliu, A. et al.), Bercelona: IEEE CS-Press, 2000, 4: 833-836.

7. Ross, A., Jain, A. K., Reisman, J., A hybrid fingerprint matcher, Pattern Recognition, 2003, 36(7): 16611673.

8. Cheng, J. G., Tian, J., Fingerprint enhancement with dyadic scale-space, Pattern Recognition Letters, 2004,25 : $1273-1284$.

9. Huttenlocher, D. F., Rucklidge, W. J., A multi-resolution technique for comparing images using the Hausdorff distance, in Proceedings of IEEE Conference of Computer Vision and Pattern Recognition, IEEE CS-Press, 1993, 705-706.

10. Luo, X. P., Tian, J., Knowledge based fingerprint image enhancement, in Proceedings of 15th International Conference on Pattern Recognition (eds. Sanfeliu, A. et al.), Bercelona: IEEE CS-Press, 2000, 4: 783-786.

11. Farina, A., Kovacs-Vajna, Z. M., Leone, A., Fingerprint minutiae extraction from skeletonized binary images, 
Pattern Recognition, 1999, 32: 877-889.

12. Fasulo, D., An analysis of Recent Work on Clustering Algorithms, University of Washington Technical Report, UW-CSE01-03-02, 1999.

13. Feng, J. F., Shi, Q. Y., Active center point-based affine matching method (in Chinese), Pattern Recognition and Intelligence, 2000, 13(3): 266-270.

14. Maio, D., Maltoni, D., Cappelli, R. et al., FVC2002: second fingerprint verification competition, in Proceedings of the16th International Conferences on Pattern Recognition (eds. Kasturi, R. et al.), Quebec: IEEE CS-Press, 2002, 3, 811-814.

15. He, Y. L., Tian, J., Luo, X. P. et al., Image enhancement and minutiae matching in fingerprint verification, Pattern Recognition Letters, 2003, 24(9-10): 1349-1360.

16. Watson, I., Wilson, C. L., NIST special database 4, fingerprint database, NIST Technical Report, 1992.

17. Watson, I., NIST special database 14, fingerprint database, NIST Technical Report, 1992.

18. Watson, I., NIST special standard reference database 24, NIST digital video of live-scan fingerprint database, NIST Technical Report, 1998.

19. Maio, D., Maltoni, D., Cappelli, R. et al., FVC2000: fingerprint verification competition, IEEE Transactions on Pattern Analysis and Machine Intelligence, 2002, 24(3): 402-412.

20. Willis, A. J., Myers, L., A cost-effective fingerprint recognition systems for use with low-quality prints and damaged fingerprints, Pattern Recognition, 2001, 34(2): 255-270.

21. Tico, M., Kuosmanen, P., Fingerprint matching using an orientation-based minutia descriptor, IEEE Transactions on Pattern Analysis and Machine Intelligence, 2003, 25(8): 1009-1014. 